\title{
Influences of nonsolvent on the morphologies and electrochemical properties of carbon nanofibres from electrospun polyacrylonitrile nanofibres
}

\author{
YULAI ZHAO*, ZHUANG ZHAO, JIAO GAO, XIANCAI JIANG, LI SHAO, HONGMEI LI and \\ LINXI HOU \\ Department of Materials-Oriented Chemical Engineering, School of Chemical Engineering, Fuzhou University, \\ Fuzhou 350116, China \\ *Author for correspondence (yulai_zhao@fzu.edu.cn)
}

MS received 10 December 2016; accepted 24 May 2017; published online 2 February 2018

\begin{abstract}
The influences of nonsolvent on the morphologies and electrochemical properties of carbon nanofibres (CNFs) obtained via pre-oxidation and carbonization of electrospun polyacrylonitrile (PAN) nanofibres were mainly studied. Volatile methanol $(\mathrm{MeOH})$ and acetonitrile $(\mathrm{MeCN})$ were introduced into PAN solutions as the nonsolvent for PAN, which may produce porous structures via inducement of phase separation. The morphologies of the prepared nanofibres were observed via scanning electron microscopy. It was found that PAN nanofibres possessed corrugated and rough surfaces. PAN fibres obtained in the presence of nonsolvent showed larger diameters and wider distributions than those obtained without nonsolvent. After thermal treatments, inter-bonded CNFs were prepared. The structures of CNFs were confirmed by X-ray diffraction and Raman spectrometry. Then the electrochemical properties of CNFs were examined by an electrochemical method in a threeelectrode system. Based on chronopotentiometry, CNFs exhibited the highest capacity up to $198 \mathrm{~F} \mathrm{~g}^{-1}$ at current density of $3 \mathrm{~A} \mathrm{~g}^{-1}$. The influence rule of nonsolvents on the morphology and capacities of CNFs was summarized and interpreted.
\end{abstract}

Keywords. Electrospinning; inter-bonded carbon nanofibres; nonsolvent effect; supercapacitor.

\section{Introduction}

Supercapacitors, namely electrochemical capacitors, as novel energy storage devices, have been widely applied in consumer electronics, portable systems, memory back-up systems, etc. Of late, supercapacitors have been receiving intense interest due to their high power density, long cycle life, charge-discharge velocity and low cost [1-4]. Supercapacitors include two types: faradaic pseudocapacitors and electric double-layer capacitors (EDLCs). Since the scale applications of the former one are limited due to poor cycling stability, high cost and so on, EDLC is the main type of supercapacitors. EDLC works by adsorbing electrolyte ions on the surface of electrode material, which is the key component for EDLC [2,5].

Many porous carbons have been widely used as electrode materials for supercapacitors due to their advantages, including large specific surface area, reasonable conductivity, controllable pore structure and low cost [2,6-8]. Among various carbon materials, electrospun carbon nanofibres (CNFs) have attracted wide attention as a promising candidate for electrode materials of supercapacitors because CNFs have adjustable and tailored chemical composition, morphology, pore structure and functionality except for the high specific surface area, thermal-chemical stability and high conductivity $[2,9,10]$. Capacity, which is a vital parameter for supercapacitor, mainly depends on the pore structure and conductivity as well as surface specific area of electrode material.

Many efforts have been spared to further increase the capacity. Kim and Yang [5] and Kim et al [11] prepared porous CNFs with micro- and meso-porosity created by tetraethyl orthosilicate (TEOS) or polyphenylsilane, which was incorporated into polyacrylonitrile (PAN) nanofibres. It was found that addition of Si-based compounds caused a notable increase of capacity. To further improve the capacitance performance, Kim et al [6] introduced graphene with excellent electrical conductivity into CNF. The capacity of resultant CNFgraphene is two times higher than that of pristine CNFs. Ma et al [9] reported the preparation of porous CNFs derived from a phenolic material, with a capacity of $171 \mathrm{~F} \mathrm{~g}^{-1}$ at $5 \mathrm{mV} \mathrm{s}^{-1}$. One weakness of CNFs is the high internal resistance for charge transfer, which impairs the performance of corresponding capacitors. This weakness could be enhanced by introducing redox-active transition metal oxides into the CNFs, including $\mathrm{RuO}_{2}, \mathrm{MnO}_{2}, \mathrm{NiO}_{x}$, etc. Yang et al [12] obtained $\mathrm{RuO}_{2}-\mathrm{CNF}$ composites with hollow structures through electrospinning of solutions containing PAN and poly(methyl methacrylate) as well as ruthenium acetylacetonate and subsequent thermal treatments. CNFs grafted with $\mathrm{RuO}_{2}$ on the surface were prepared via the hydrothermal deposition technique by Chuang et al [13]. The capacity of supercapacitors exhibited remarkable increase with the 
incorporation of $\mathrm{RuO}_{2}$, which simultaneously increased the cost of CNFs.

The fibrous morphology also has an influence on the electrochemical properties. The electrospun CNFs have nonwoven morphology in which the fibres are relatively independent of each other without sufficient contact, which results in high contact resistance. Inter-connected or inter-bonded CNFs have been reported by Niu et al [2]. The inter-connected CNFs were obtained with a higher capacity (up to $221 \mathrm{~F} \mathrm{~g}^{-1}$ ) via bicomponent electrospinning than those via conventional electrospinning. Lu et al [3] reported the preparation of cross-linked porous CNFs by electrospinning PAN solutions containing tripolycyanamide. The obtained CNFs showed high capacity and excellent cycling stability. Furthermore, solvent properties would influence the electrospun fibres. Yu et al [14] and Ou et al [15] prepared porous PAN fibres by electrospinning PAN-N, N-dimethylformamide (DMF)-water solutions and PAN-DMF-dimethyl sulphoxide solutions, based on nonsolvent-induced phase separation mechanism. In addition, several other porous polymer fibres were obtained by the aforementioned mechanism $[16,17]$.

PAN nanofibres are currently the most important precursors for the preparation of CNFs. In the present work, the influences of nonsolvents methanol and acetonitrile on the morphologies of PAN nanofibres were studied. Further, the influences of nonsolvent on the morphologies and electrochemical properties of CNFs derived from electrospun PAN fibres were investigated, which have been seldom reported in previous reports.

\section{Experimental}

\subsection{Materials}

PAN $\left(M_{\mathrm{w}} \sim 150000\right)$ was provided by Shanghai Macklin Biochemical Co., Ltd. N, N-dimethylformamide (DMF), methanol and acetonitrile $(\mathrm{MeCN})$ were provided by Tianjin Zhiyuan Chemical Reagent Co. Ltd. All the chemicals were used as received without purification.

\subsection{Preparation of CNFs}

A certain weight of PAN was dissolved in DMF-based solvent containing methanol or acetonitrile as nonsolvent for PAN. The mass concentrations of all solutions are maintained constant. Solutions containing varying contents of methanol or acetonitrile were prepared to investigate the effects of the nonsolvent.

PAN nanofibres were fabricated via electrospinning technique. The device consists of a high voltage power supply (DN-P303-1ACF0, Dongwen High Voltage), a syringe pump (TJ-3A, LongerPump) and a grounded rolling collector covered with aluminium-foil paper. The electrospinning was operated under a voltage of $12 \mathrm{kV}$ in room atmosphere for about $1 \mathrm{~h}$. The electropsun PAN fibres were pre-oxidized in air atmosphere at $250^{\circ} \mathrm{C}$ for $2 \mathrm{~h}$. Then the pre-oxidized fibres were carbonized at $800^{\circ} \mathrm{C}$ in $\operatorname{argon}$ atmosphere for $1 \mathrm{~h}$ at a heating rate of $5^{\circ} \mathrm{C} \min ^{-1}$ to produce PAN-based CNFs.

\subsection{Characterizations}

The morphologies of the samples were observed using a scanning electron microscope (SEM, Hitati SU-8010, Japan). Average diameters of fibres were analysed by Nano Measurer 1.2 based on at least 15 data for each sample. The structure was determined by X-ray diffraction (XRD, X'Pert PRO, PANalytical, Netherlands) and Raman spectra (inVia Reflex, Renishawn, England).

The samples for electrochemical tests were made by putting $\sim 3 \mathrm{mg}$ of nonwoven CNF between two pieces of nickel foams $(1.5 \mathrm{~cm} \times 1.5 \mathrm{~cm})$ acting as current collector. Then, the samples were compressed under a pressure of $10 \mathrm{MPa}$ for $10 \mathrm{~min}$. The cyclic voltammetry $(\mathrm{CV})$ and chronopotentiometry $(\mathrm{CP})$ curves of the samples were recorded on an electrochemical workstation (CHI660E, Shanghai). The tests were carried out in a three-electrode system using $\mathrm{Ag}-\mathrm{AgCl}$ as the reference electrode and $\mathrm{Pt}$ foil as the counter-electrode; $6.0 \mathrm{M}$ of $\mathrm{KOH}$ solution was used as the electrolyte for all electrochemical measurements.

\section{Results and discussion}

\subsection{Morphologies of PAN fibres and CNFs}

Firstly, PAN nanofibres were prepared by electrospinning PAN solutions in different mixed solvents. To ensure complete dissolution of PAN, an intensive polar solvent, methanol, was chosen, and acetonitrile was chosen as the second solvent to mix with DMF. In addition, percentage of methanol in mixed solvent was lower than that of acetonitrile. The detailed conditions are summarized in table 1. Morphologies of the obtained PAN fibres were observed via SEM. The results are collected in figure 1, which shows that uniform fibres with average diameter of $0.41 \mu \mathrm{m}$ are obtained from the solution in pure DMF. On increasing methanol content in mixed solvent, the diameter of fibres showed a slight increase. Interestingly, the diameter increased notably from 0.41 to $0.96 \mu \mathrm{m}$ with the increase of acetonitrile percent as summarized in table 1 . Furthermore, fibres with broader diameter distributions were obtained with the addition of acetonitrile as shown in figure $2 \mathrm{a}$. It should be noted that the absence of more experiments with the same percent of acetonitrile and methanol is attributed to the notable difference in the dissolving capacity of PAN.

It was observed that all the fibres have corrugated and rough surfaces might be attributed to the shrinkage of the fibres in the electrospinning process caused by the solvent evaporation. The composition of mixed solvents showed negligible influence on the surface morphology.

Through stabilization in air and subsequent carbonization in $\mathrm{Ar}, \mathrm{CNFs}$ were prepared and the morphologies were 
Table 1. Results of PAN fibres and CNFs from solutions in different mixed solvents. ${ }^{\mathrm{a}}$

\begin{tabular}{lccccc}
\hline & & & & & \multicolumn{2}{c}{ CNFs } \\
\cline { 5 - 6 } Run & Methanol (vol\%) & $\begin{array}{c}\text { Acetonitrile } \\
(\text { vol } \%)\end{array}$ & $\begin{array}{c}\text { PAN fibres } \\
D_{\text {average }}(\mu \mathrm{m})\end{array}$ & $D_{\text {average }}(\mu \mathrm{m})$ & $I_{\mathrm{D}} / I_{\mathrm{G}}^{\mathrm{b}}$ \\
\hline 1 & 0 & 0 & 0.41 & 0.30 & 1.103 \\
2 & 2 & 0 & 0.41 & - & - \\
3 & 4 & 0 & 0.44 & 0.45 & 1.107 \\
4 & 6 & 0 & 0.47 & 0.39 & 1.115 \\
5 & 0 & 4 & 0.48 & 0.33 & 1.036 \\
6 & 0 & 12 & 0.91 & 0.74 & 1.045 \\
7 & 0 & 20 & 0.96 & 0.67 & 0.977 \\
\hline
\end{tabular}

a Other conditions: room temperature, concentration $150 \mathrm{mg} \mathrm{ml}^{-1}$, working voltage $=12 \mathrm{kV}$, feed rate $=0.5 \mathrm{ml} \mathrm{h}^{-1}$ and the distance between the end of spinneret and collector is $15 \mathrm{~cm}$.

${ }^{\mathrm{b}}$ Calculated based on Raman spectra.
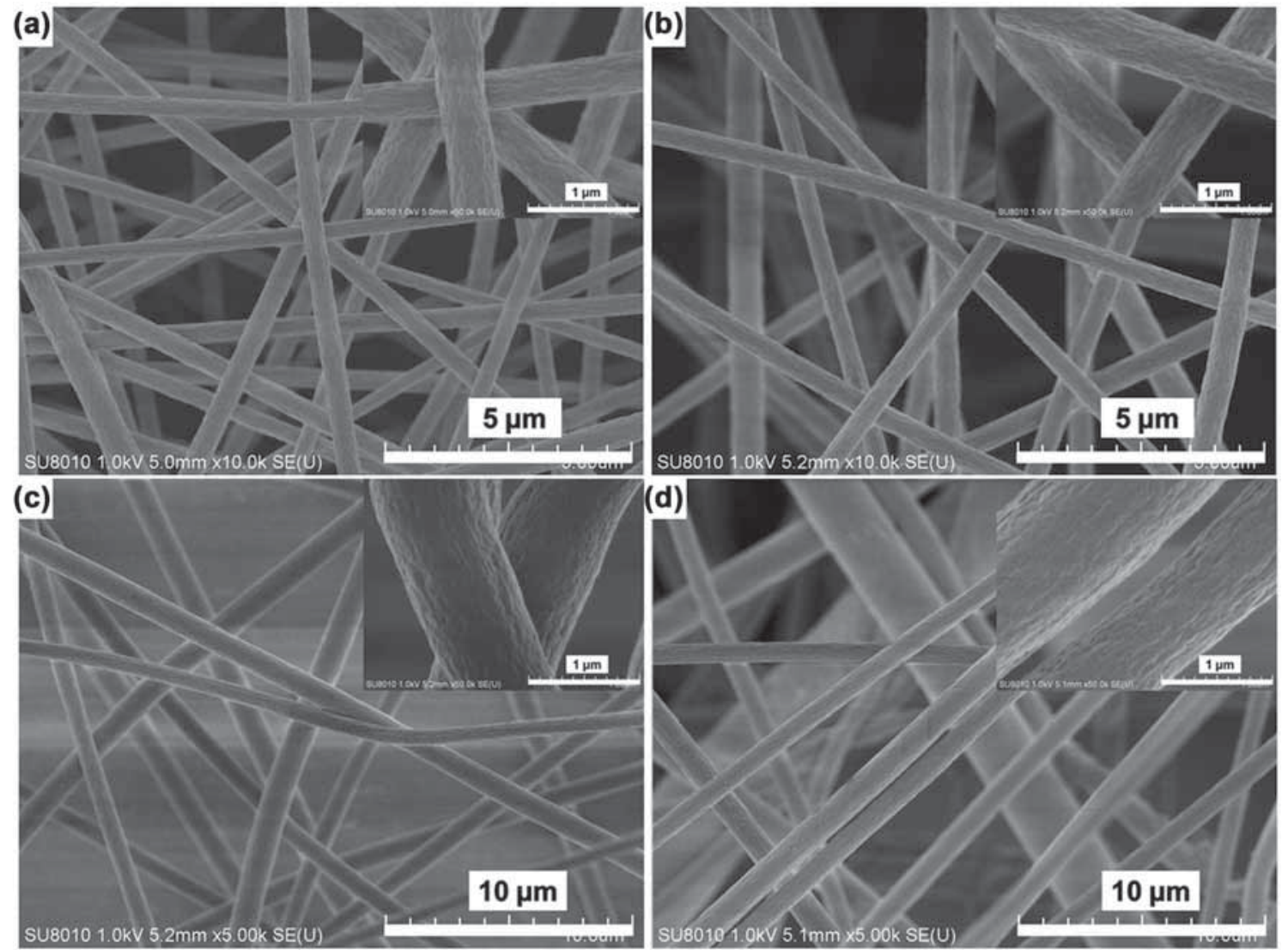

Figure 1. SEM images of PAN fibres electrospun at $12 \mathrm{kV}$ from solutions in (a) pure DMF and mixed solvents with (b) methanol (6 vol\%), (c) acetonitrile (12 vol\%) and (d) acetonitrile (20 vol\%).

observed using the SEM, as shown in figure 3. It was found that bended CNFs were bonded to the adjacent ones. As a result, a fibrous net with irregular holes was obtained, which may endow the CNFs with new electrochemical properties. The CNF from pure DMF solution showed a diameter of $\sim 0.30 \mu \mathrm{m}$. CNFs have smaller diameters than those of corresponding PAN fibres due to physical and chemical reactions during thermal treatments. Similar to PAN fibres, diameter of CNFs become larger when the content of acetonitrile increases. The detailed data are summarized in table 1 and figure $2 b$.

\subsection{Structures via XRD and Raman spectra}

The structures of CNFs were studied via XRD and Raman spectroscopy. Figure 4a shows typical XRD patterns. 

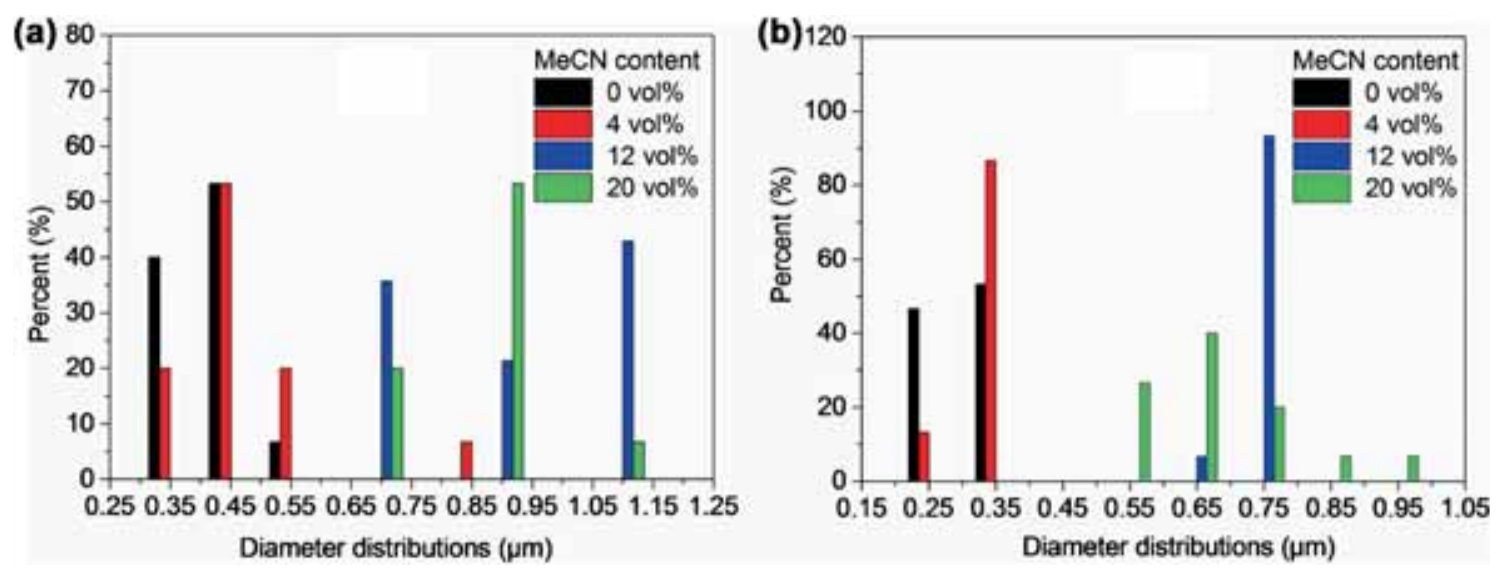

Figure 2. Diameter distributions of (a) prepared PAN fibres and (b) CNF obtained from solutions in different solvents.
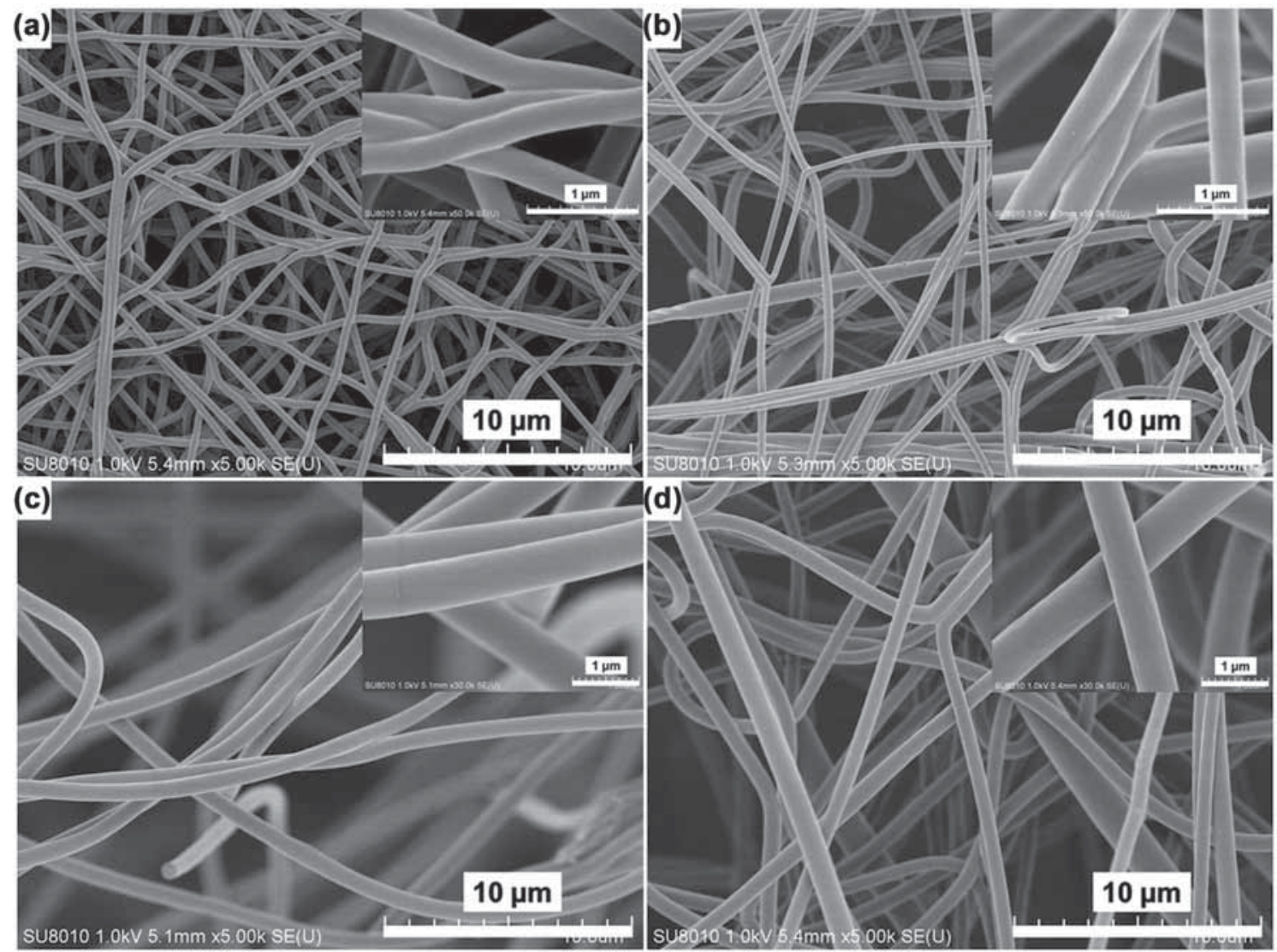

Figure 3. SEM images of CNFs evolved from PAN fibres electrospun from solutions in (a) pure DMF and mixed solvents with (b) methanol (6 vol\%), (c) acetonitrile (12 vol\%) and (d) acetonitrile (20 vol\%).

The broad peaks appearing between $20^{\circ}$ and $30^{\circ}$ for all samples can be assigned to $d_{(002)}$ layers of graphitic crystallites. In addition, relatively weak and broad peaks appearing at $\sim 44^{\circ}$ might be assigned to $d_{(101)}$ layers, corresponding to the graphite basal plane. The broad shape of the peaks indicate amorphous phase in the CNFs $[2,18]$. The peaks of CNFs appearing at higher $2 \theta$ than those in references $[2,18]$ indicate that the prepared CNFs possess smaller layer distance according to the Bragg equation. It was found that the CNFs obtained in the mixed solvents showed stronger diffraction peaks, implying that these CNFs possess higher crystallinity.

The structure of CNFs was further studied via Raman spectra, which exhibit two characteristic peaks of CNFs as shown in figure 4b. The two peaks appearing at 1345 and $1580 \mathrm{~cm}^{-1}$, assigned to $\mathrm{D}$ peak from amorphous structures of carbon and G peak from graphitic structures of carbon, respectively [2,5]. 

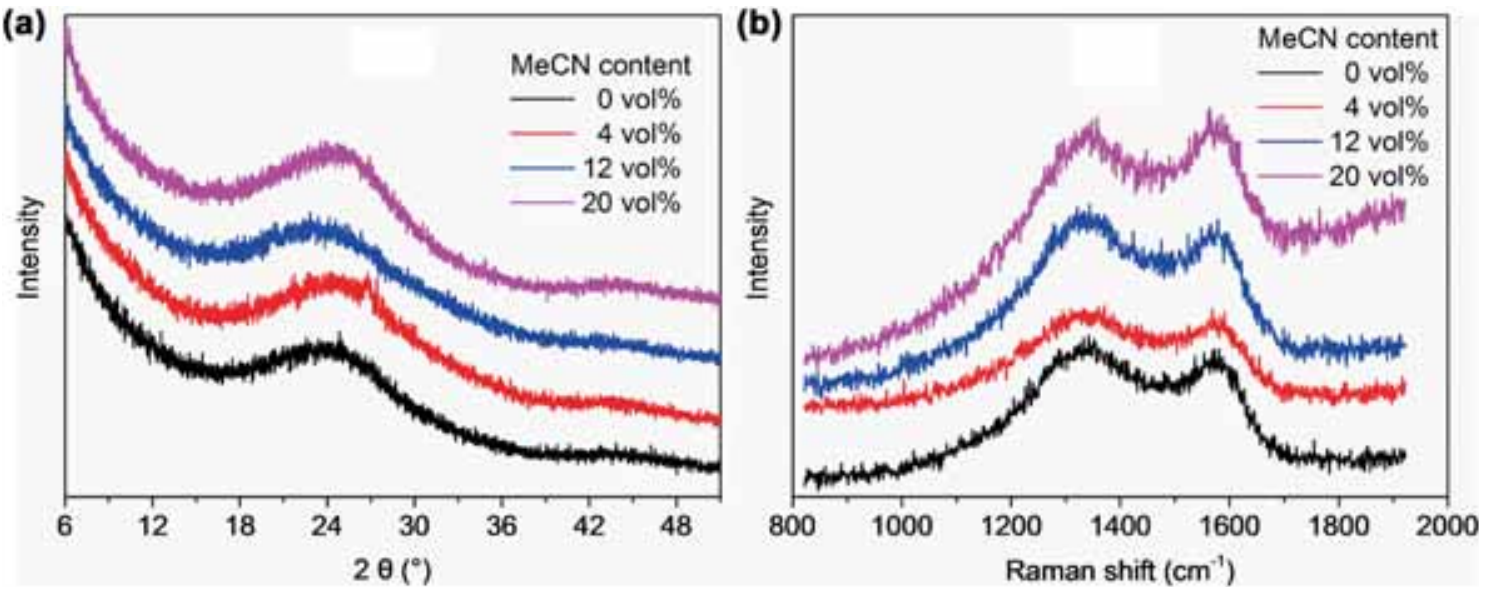

Figure 4. (a) XRD patterns and (b) Raman spectra of carbon nanofibres evolved from PAN fibres in different solutions.
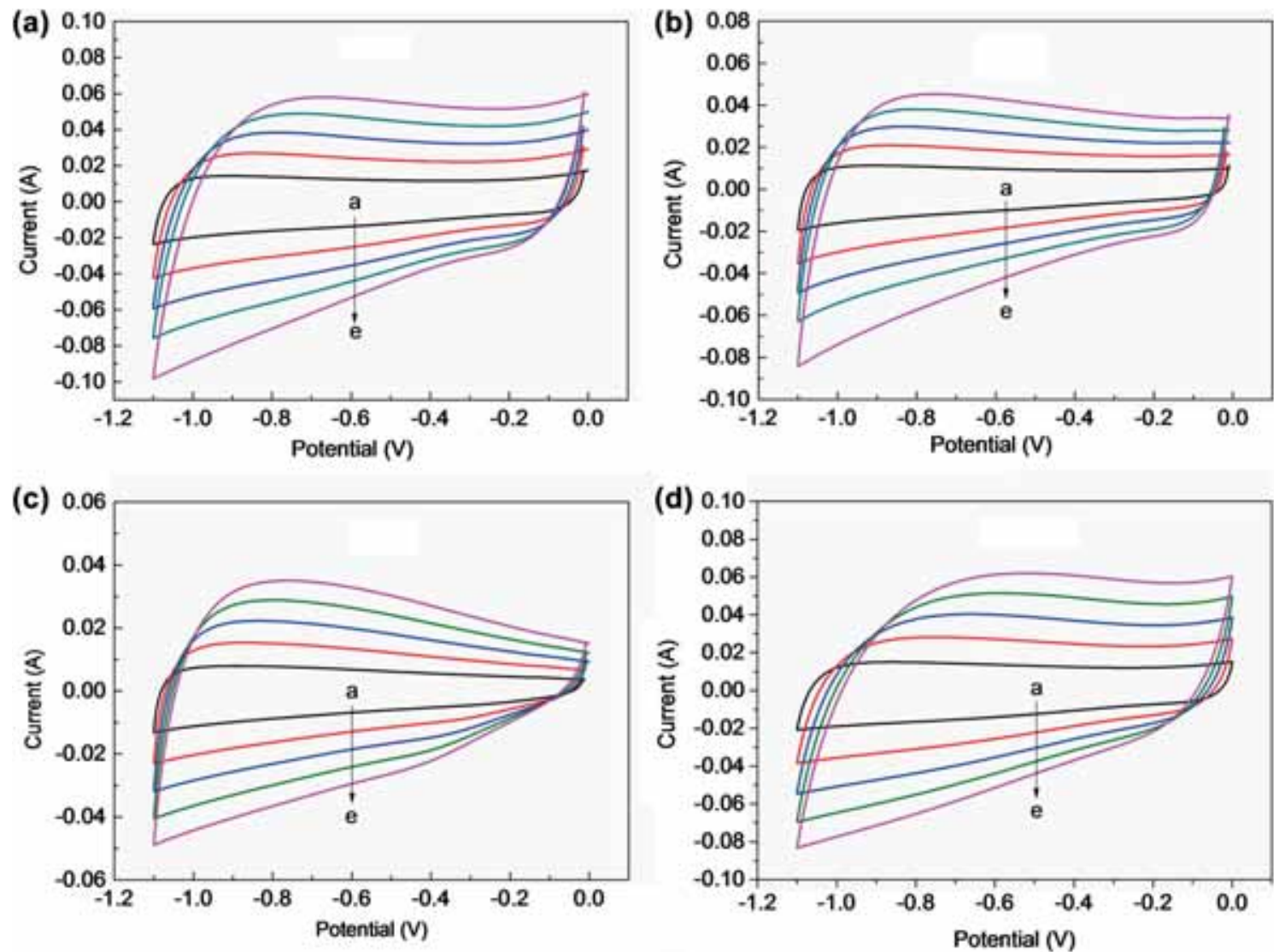

Figure 5. CV curves of CNFs evolved from PAN fibres electrospun from solutions containing acetonitrile: (a) 0 vol $\%$, (b) $4 \mathrm{vol} \%$ and (c) $20 \mathrm{vol} \%$ and (d) methanol (4 vol\%) (a $\rightarrow$ e: $20,40,60,80$ and $\left.100 \mathrm{mV} \mathrm{s}^{-1}\right)$.

The intensity ratio $R\left(R=I_{\mathrm{D}} / I_{\mathrm{G}}\right)$ data of CNFs collected in table 1 indicate that $\mathrm{MeOH}$ and $\mathrm{MeCN}$ show different influences on the structure of CNF. With the increase of MeCN content, $R$-value reduced, implying that CNFs with better graphitic structure were obtained, which is consistent with the XRD results $[2,19]$. However, in the case of $\mathrm{MeOH}$, reverse change was observed. It might be deduced that $-\mathrm{CN}$ group of $\mathrm{MeCN}$ is favourable to obtain PAN fibres with good alignment.

\subsection{Supercapacitive performances of $C N F s$}

The electrochemical performances of CNFs were studied with the three-electrode system to evaluate its possibility of use 

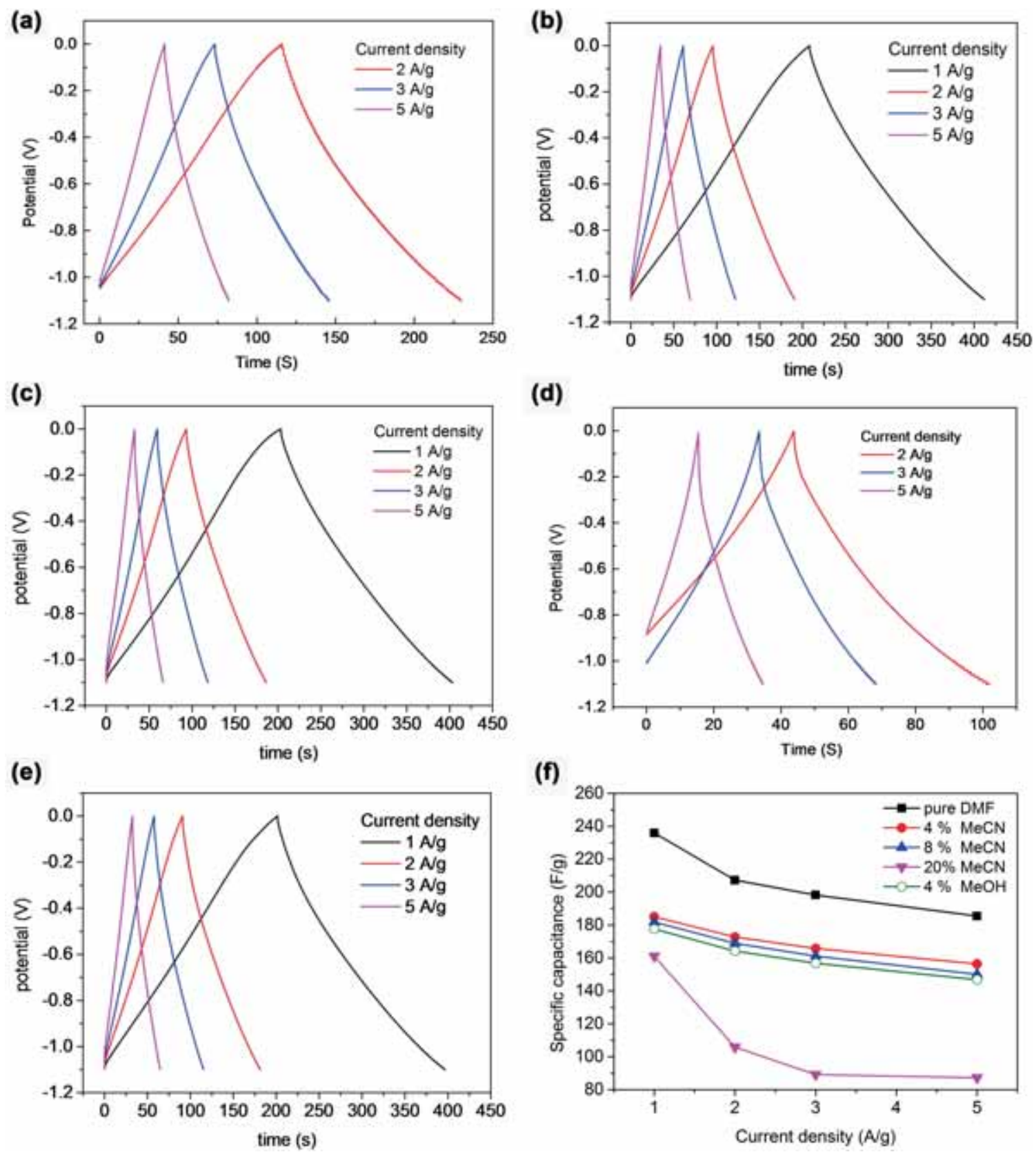

Figure 6. Galvanostatic charge-discharge curves of CNFs evolved from PAN fibres electrospun from solutions containing acetonitrile: (a) $0 \mathrm{vol} \%$, (b) $4 \mathrm{vol} \%$, (c) $8 \mathrm{vol} \%$, (d) $20 \mathrm{vol} \%$, (e) methanol (4 vol\%) and (f) the calculated specific capacities.

as supercapacitor. As shown in figure 5, the CV curves of the samples at varying scan rates show nearly rectangular shape, which is typical for supercapacitors. At high scan rates, the rectangular shape is distorted a little bit. This is mainly because at high scan rate, time is not sufficient for electrolytes to reach the pore structures on the surface. Furthermore, at a high $\mathrm{MeCN}$ content in the mixed solvent, the $\mathrm{CV}$ curves of obtained CNFs exhibit notable deviations from rectangular shape (figure 5c), implying the less-than-ideal EDLC behaviour. This might be attributed to pseudo-faradic reactions of redox active sites in the CNFs obtained in the presence of high content of $\mathrm{MeCN}$. It is possible that the $-\mathrm{CN}$ group of residual $\mathrm{MeCN}$ may participate in the preoxidation and carbonization. For all samples, the specific capacitances estimated from CV curves showed decreasing trends with the increase of scan rate, in accordance with the previous reports $[9,18]$. CNF samples obtained in the presence of $\mathrm{MeCN}$ exhibited decreased specific capacitances with the increase of $\mathrm{MeCN}$ content. In addition, the $\mathrm{CV}$ curves of CNFs obtained with $\mathrm{MeOH}$ (figure 5d) as the nonsolvent showed shapes similar to those obtained in the presence of $\mathrm{MeCN}$. 
Galvanostatic charge-discharge curves of obtained CNF samples at different current densities are summarized in figure 6. All the charge-discharge curves obtained at other current densities all showed highly symmetrical triangular shapes, indicating excellent reversibility and conductivity of the electrode [20]. The specific capacitances $\left(C_{\mathrm{g}}\right)$ were calculated as shown in figure $6 \mathrm{~d}$ using the equation $C_{\mathrm{g}}=\mathrm{It} / \mathrm{m} \Delta \mathrm{V}$, where $I$ and $t$ are the discharge current (A) and discharge time (s), respectively; $m$ is the weight of $\mathrm{CNF}$ ( $\mathrm{g}$ ) and $\Delta V$ is potential difference (V) [9]. It was found that CNF obtained in the presence of $\mathrm{MeCN}$ showed lower specific capacitance than that obtained in pure DMF, which is consistent with results from CV curves. The possible reason is that the CNFs were obtained with a larger average diameter in the presence of $\mathrm{MeCN}$, which results in lower specific surface area, which is crucial for the capacitance of electrodes since the specific capacitance of CNF is generally in direct proportion to its specific surface area. Consequently, the CNFs obtained in mixed solvents showed decreased capacitance. The CNF obtained in the presence of $\mathrm{MeOH}$ also showed a lower capacitance than that in pure DMF, which is similar to the case using $\mathrm{MeCN}$ as the nonsolvent. Moreover, with the same percent of nonsolvent, the capacity of CNF obtained with $\mathrm{MeOH}$ is lower than that with $\mathrm{MeCN}$.

The specific capacitance of CNF obtained in DMF is $235 \mathrm{~F} \mathrm{~g}^{-1}$ at density of $1 \mathrm{~A} \mathrm{~g}^{-1}$, which is close to the results by Niu et al [2] and Zhao et al [21]. This might be attributed to the cross-linked structures or interconnections of CNFs, which endow the CNF with excellent conductivity and high charge transfer efficiency [2,3]. Also, with the increase of current density, the specific capacitance decreased. The CNFs obtained with 0,4 and $20 \%$ of $\mathrm{MeCN}$ showed capacities of 185,156 and $88 \mathrm{~F} \mathrm{~g}^{-1}$ at a current density of $5 \mathrm{~A} \mathrm{~g}^{-1}$, preserving 78,84 and $55 \%$ of the capacities measured at $1 \mathrm{~A} \mathrm{~g}^{-1}$, respectively.

The cycling stability of obtained CNFs was tested by the galvanostatic charge-discharge method with a current density of $3 \mathrm{~A} \mathrm{~g}^{-1}$. Almost no reduction of capacity was observed, indicating excellent stability within 300 charge-discharge circles though very little black sediment was observed in the electrolyte after the circling test, which might be due to the absence of binder.

\section{Conclusions}

CNFs with inter-bonded structures were obtained via preoxidation and carbonization of electrospun PAN nanofibres. Nonsolvents methanol and acetonitrile showed notable influence on the fibrous morphologies and electrochemical performance. The diameters of PAN nanofibres increased with the content of acetonitrile in polymer solution. The structure of CNFs was confirmed by XRD and Raman spectrometry. The highest specific capacity of CNFs reached $198 \mathrm{~F} \mathrm{~g}^{-1}$ at a current density of $3 \mathrm{~A} \mathrm{~g}^{-1}$. Considering the effect of nonsolvent on the morphology of CNFs, the reduction of capacity with the addition of nonsolvent might be attributed to the decrease of specific surface area caused by increased diameter. A possible conclusion could be deduced that the addition of nonsolvent that produces porous structures but simultaneously increases the diameters is not rational to enhance the electric capacity of CNFs.

\section{Acknowledgements}

We would like to thank the Science and Technology Program of Education Department of Fujian Province (JA15065), Natural Science Foundation of Fujian Province (2017J01695), and Scientific Research Starting Foundation of Fuzhou University (510126) for financial supports.

\section{References}

[1] Kim B H, Yang K S and Woo H G 2011 Electrochem. Commun. 131042

[2] Niu H, Zhang J, Xie Z, Wang X and Lin T 2011 Carbon 49 2380

[3] Lu J J, Ying Z R, Liu X D and Zhao S S 2015 Acta Phys. Chim. Sin. 312099

[4] Wu X M, Wang Q G, Zhang W Z, Wang Y and Chen W X 2016 J. Mater. Sci. 517731

[5] Kim B-H and Yang K S 2014 J. Electroanal. Chem. 71492

[6] Kim S Y, Kim B-H, Yang K S and Oshida K 2012 Mater. Lett. 87157

[7] Wang Y Z, Liu Y, Liu W, Zhang G X, Liu G W, Chen H Y et al 2016 J. Alloys Compd. 677105

[8] Yi H T, Zhu Y Q, Chen X Y and Zhang Z J 2015 J. Alloys Compd. 649851

[9] Ma C, Song Y, Shi J, Zhang D, Zhong M, Guo Q et al 2012 Mater. Lett. 76211

[10] Mao X, Hatton T A and Rutledge G C 2013 Curr. Org. Chem. 171390

[11] Kim B-H, Yang K S and Woo H G 2012 Electrochim. Acta 59 202

[12] Yang K S, Kim C H and Kim B-H 2015 Electrochim. Acta 174 290

[13] Chuang C-M, Huang C-W, Teng H and Ting J-M 2012 Compos. Sci. Technol. 721524

[14] Yu X, Xiang H, Long Y, Zhao N, Zhang X and Xu J 2010 Mater. Lett. 642407

[15] Ou Y, Wu Q, Wan L, Yang H and Xu Z 2013 Acta Polym. Sin. 248015

[16] Katsogiannis K A G, Vladisavljevic G T and Georgiadou S 2015 Eur. Polym. J. 69284

[17] Wei Z, Zhang Q, Wang L, Wang X, Long S and Yang J 2013 Colloid Polym. Sci. 2911293

[18] Wang G, Pan C, Wang L, Dong Q, Yu C, Zhao Z et al 2012 Electrochim. Acta 6965

[19] Karthikeyan K K and Biji P 2016 Microporous Mesoporous Mater. 224372

[20] Zhang X-B, Chen M-H, Zhang X-G and Li Q W 2010 Acta Phys. Chim. Sin. 263169

[21] Zhao H, Wang L, Jia D, Xia W, Li J and Guo Z 2014 J. Mater. Chem. A 29338 